Pacific Journal of Mathematics

A SIMPLE FORMULA FOR CONDITIONAL WIENER 


\section{A SIMPLE FORMULA FOR CONDITIONAL WIENER INTEGRALS WITH APPLICATIONS}

\section{Chull Park and David Skoug}

Yeh's inversion formula for conditional Wiener integrals is very complicated to apply when the conditioning function is vector-valued. This paper gives a very simple formula for such integrals. In particular, we express the conditional Wiener integral directly in terms of an ordinary (i.e., nonconditional) Wiener integral. Using this new formula, it is very easy to generalize the Kac-Feynman formula and also to obtain a Cameron-Martin type translation theorem for general conditional Wiener integrals.

1. Introduction. Consider the Wiener measure space $\left(C[0, T], F^{*}\right.$, $m_{w}$ ) where $C[0, T]$ is the space of all continuous functions $x$ on $[0, T]$ vanishing at the origin. For each partition $\tau=\tau_{n}=\left\{t_{1}, \ldots, t_{n}\right\}$ of $[0, T]$ with $0=t_{0}<t_{1}<\cdots<t_{n}=T$, let $X_{\tau}: C[0, T] \rightarrow \mathbf{R}^{n}$ be defined by $X_{\tau}(x)=\left(x\left(t_{1}\right), \ldots, x\left(t_{n}\right)\right)$. Let $\mathscr{B}^{n}$ be the $\sigma$-algebra of Borel sets in $\mathbf{R}^{n}$. Then a set of the type

$$
I=\left\{x \in C[0, T]: X_{\tau}(x) \in B\right\} \equiv X_{\tau}^{-1}(B), \quad B \in \mathscr{B}^{n},
$$

is called a Wiener interval (or a Borel cylinder). It is well known that

$$
m_{w}(I)=\int_{B} K(\tau, \vec{\xi}) d \vec{\xi}
$$

where

$$
\begin{array}{r}
K(\tau, \vec{\xi})=\left\{\prod_{j=1}^{n} 2 \pi\left(t_{j}-t_{j-1}\right)\right\}^{-1 / 2} \exp \left\{-\frac{1}{2} \sum_{j=1}^{n} \frac{\left(\xi_{j}-\xi_{j-1}\right)^{2}}{t_{j}-t_{j-1}}\right\} \\
\vec{\xi}=\left(\xi_{1}, \ldots, \xi_{n}\right), \text { and } \quad \xi_{0}=0 .
\end{array}
$$

$m_{w}$ is a probability measure defined on the algebra $\mathscr{F}$ of all Wiener intervals and $m_{w}$ is extended to the Caratheodory extension $\mathscr{F}^{*}$ of $\mathscr{F}$. Let $\mathscr{F}_{\tau}$ be the $\sigma$-algebra generated by the set $\left\{X_{\tau}^{-1}(B): B \in \mathscr{B}^{n}\right\}$ with $\tau$ fixed. Then, by the definition of conditional expectation (see Tucker 
[6] and Yeh [8]), for each Wiener integrable function $F(x)$

$$
\begin{aligned}
\mu_{\tau}(B) & \equiv \int_{X_{\tau}^{-1}(B)} F(x) m_{w}(d x)=\int_{X_{\tau}^{-1}(B)} E\left(F \mid \mathscr{F}_{\tau}\right) m_{w}(d x) \\
& =\int_{B} E\left(F(x) \mid X_{\tau}(x)=\vec{\xi}\right) P_{X_{\tau}}(d \vec{\xi}), \quad B \in \mathscr{B}^{n}
\end{aligned}
$$

where $P_{X_{\tau}}(B)=m_{w}\left(X_{\tau}^{-1}(B)\right)$, and $E\left(F(x) \mid X_{\tau}(x)=\vec{\xi}\right)$ is a Borel measurable function of $\vec{\xi}$ which is unique up to Borel null sets in $\mathbf{R}^{n}$. According to Yeh [8], the conditional expectation $E\left(F(x) \mid X_{\tau}(x)=\vec{\xi}\right)$ can be found by the Fourier transform

$$
\begin{aligned}
& E\left(F(x) \mid X_{\tau}(x)=\vec{\xi}\right) \\
& \quad=\left[\frac{d p_{X_{\tau}}}{d m_{L}}(\vec{\xi})\right]^{-1}(2 \pi)^{-n} \int_{\mathbf{R}^{n}} e^{-i\langle O, \bar{\xi}\rangle} E\left[e^{i\left\langle\vec{U}, \vec{X}_{t}\right\rangle} F\right] m_{L}(d \vec{U})
\end{aligned}
$$

where $m_{L}$ is Lebesgue measure on $\mathbf{R}^{n}$. Using the above inversion formula (changing the conditional expectation into non-conditional expectation) for the special case $X_{\tau}(x)=x(T)$, i.e., $\tau$ consists of the endpoint only, Yeh $[9,10]$ obtains very useful results including the Kac-Feynman integral equation and the conditional Cameron-Martin translation theorem. However, Yeh's inversion formula (1.4) is very difficult and complicated to apply for general $\tau$ 's. Some results are obtained by Chang and Chang [3] for the latter case using Yeh's formula (1.4), but these results are somewhat limited in scope and the calculations are quite lengthy in general.

The main purpose of this paper is to develop a new simple formula for the conditional Wiener integral when the conditioning function is vector-valued and then to obtain a general form of the Kac-Feynman formula and a general conditional Cameron-Martin translation theorem.

We thank the referee for very helpful suggestions, which have led to the present version of this paper.

2. A simple formula for conditional Wiener integrals. For a given partition $\tau=\tau_{n}$ of $[0, T]$ and $x \in C[0, T]$, define the polygonal function $[x]$ on $[0, T]$ by

$$
\begin{aligned}
{[x](t)=x\left(t_{j-1}\right)+\frac{t-t_{j-1}}{t_{j}-t_{j-1}}\left(x\left(t_{j}\right)-x\left(t_{j-1}\right)\right), } & \\
& t_{j-1} \leq t \leq t_{j}, j=1, \ldots, n .
\end{aligned}
$$


Similarly, for each $\vec{\xi}=\left(\xi_{1}, \ldots, \xi_{n}\right) \in \mathbf{R}^{n}$, define the polygonal function $[\vec{\xi}]$ of $\vec{\xi}$ on $\{0, T]$ by

$$
\begin{aligned}
& {[\vec{\xi}](t)=\xi_{j-1}+\frac{t-t_{j-1}}{t_{j}-t_{j-1}}\left(\xi_{j}-\xi_{j-1}\right),} \\
& t_{j-1} \leq t \leq t_{j}, j=1, \ldots, n \text { and } \xi_{0}=0 .
\end{aligned}
$$

Then both $[x]$ and $[\vec{\xi}]$ are continuous on $[0, T]$, their graphs are line segments on each subinterval $\left[t_{j-1}, t_{j}\right]$, and $[x]\left(t_{j}\right)=x\left(t_{j}\right)$ and $[\vec{\xi}]\left(t_{j}\right)=$ $\xi_{j}$ at each $t_{j} \in \tau$.

The following theorem gives an interesting observation for the process $x(t)-[x](t)$. While the process $[x](t)$ has been widely used to approximate the Brownian motion $x(t)$, recognizing $x(t)-[x](t)$ as a Brownian bridge on each subinterval is new as far as the authors know.

Theorem 1. If $\{x(t), 0 \leq t \leq T\}$ is the standard Wiener process, then the process $\{x(t)-[x](t), 0 \leq t \leq T\}$ and $X_{\tau}(x)=\left(x\left(t_{1}\right), \ldots, x\left(t_{n}\right)\right)$ are (stochastically) independent.

Proof. In view of (2.1), we may write for $t_{j-1} \leq t \leq t_{j}$

$$
x(t)-[x](t)=\left(x(t)-x\left(t_{j-1}\right)\right)-\frac{t-t_{j-1}}{t_{j}-t_{j-1}}\left(x\left(t_{j}\right)-x\left(t_{j-1}\right)\right) .
$$

Note that $x(t)-[x](t)$ is a Brownian bridge process on $\left\{t_{j-1}, t_{j}\right]$ vanishing at $t_{j-1}$ and $t_{j}$. Thus $Y \equiv\left\{x(t)-[x](t), t_{j-1} \leq t \leq t_{j}\right\}$ is independent of the process $\left\{x(t): t \in\left[0, t_{j-1}\right] \cup\left[t_{j}, T\right]\right\}$. In particular, $Y$ is independent of $X_{\tau}(x)=\left(x\left(t_{1}\right), \ldots, x\left(t_{n}\right)\right)$. Since $j$ was arbitrary, the proof is complete.

Corollary 1. If $\{x(t), 0 \leq t \leq T\}$ is the standard Wiener process, then $\left\{x(t)-[x](t), t_{j-1} \leq t \leq t_{j}\right\}, j=1,2, \ldots, n$ are independent Brownian bridge processes.

The following theorem is one of our main results:

THEOREM 2. Let $F$ be any Wiener integrable function on $C[0, T]$. Then, for every Borel measurable set $B$ in $\mathbf{R}^{n}$,

$$
\begin{aligned}
\mu_{\mathrm{t}}(B) & \equiv \int_{X_{\tau}^{-1}(B)} F(x) m_{w}(d x) \\
& =\int_{B} E[F(x-[x]+[\vec{\xi}])] P_{X_{\mathrm{\tau}}}(d \vec{\xi}) .
\end{aligned}
$$


Proof. First assume that $F$ is the characteristic function of a Wiener measurable set $A$, i.e., $F(x)=\chi_{A}(x)$. Then

$$
\begin{aligned}
\int_{X_{\tau}^{-1}(B)} \chi_{A}(x) m_{w}(d x)=m_{w}\left(A \cap X_{\tau}^{-1}(B)\right) \\
=\int_{B} m_{w}\left(x \in A \mid X_{\tau}(x)=\vec{\xi}\right) P_{X_{\tau}}(d \vec{\xi}) \\
=\int_{B} m_{w}\left(x-[x]+[\vec{\xi}] \in A \mid X_{\tau}(x)=\vec{\xi}\right) P_{X_{\tau}}(d \vec{\xi}) .
\end{aligned}
$$

But, $x-[x]$ and $X_{\tau}(x)$ are independent by Theorem 1. Thus,

$$
\begin{aligned}
\int_{X_{\tau}^{-1}(B)} \chi_{A}(x) m_{w}(d x) & =\int_{B} m_{w}(x-[x]+[\vec{\xi}] \in A) P_{X_{\tau}}(d \vec{\xi}) \\
& =\int_{B} E\left[\chi_{A}(x-[x]+[\vec{\xi}])\right] P_{X_{\tau}}(d \vec{\xi}) .
\end{aligned}
$$

Thus, the result holds for the characteristic function of any Wiener measurable set. The general case follows by the usual arguments in integration theory.

If $F$ is a Wiener integrable function on $C[0, T]$, then from (2.4), the Radon-Nikodym derivative of $\mu_{\tau}$ with respect to $P_{X_{\mathrm{r}}}$ is

$$
\frac{d \mu_{\tau}}{d P_{X_{\tau}}}(\vec{\xi})=E[F(x-[x]+[\vec{\xi}])]
$$

for a.e. $\vec{\xi}$ in $\mathbf{R}^{n}$. But (1.3) yields

$$
\frac{d \mu_{\tau}}{d P_{X_{\tau}}}(\vec{\xi})=E\left(F(x) \mid X_{\tau}(x)=\vec{\xi}\right)
$$

for a.e. $\vec{\xi}$ in $\mathbf{R}^{n}$. Thus $E\left(F(x) \mid X_{\tau}(x)=\vec{\xi}\right)$ and $E[F(x-[x]+[\vec{\xi}])]$ are equal a.e. on $\mathbf{R}^{n}$. However, while $E\left(F(x) \mid X_{\tau}(x)=\vec{\xi}\right)$ is always Borel measurable in $\vec{\xi}$ by definition, $E[F(x-[x]+[\vec{\xi}])]$ is Lebesgue measurable in $\vec{\xi}$ but not necessarily Borel measurable as the following example shows.

EXAMPLE 1. Let $G$ be a Legesgue measurable null set in $\mathbf{R}$ that is not Borel measurable. Then the function $f(\xi)=\chi_{G}(\xi)$ is Lebesgue measurable but is not Borel measurable. Let $A=\{x \in C[0, T]: x(T) \in$ $G$. Then $m_{w}(A)=0$. Define $F: C[0, T] \rightarrow \mathbf{R}$ by $F(x)=\chi_{A}(x)$. Then $F(x)=\chi_{G}(x(T))$ and

$$
\begin{aligned}
E[F(x-[x]+[\xi])] & =E\left[\chi_{G}(x(T)-[x](T)+[\xi](T))\right] \\
& =E\left[\chi_{G}(\xi)\right]=\chi_{G}(\xi)=f(\xi) .
\end{aligned}
$$


However, if $f(\vec{\xi})$ is Lebesgue measurable on $\mathbf{R}^{n}$, then there exists a Borel measurable function $\hat{f}(\vec{\xi})$ such that $\hat{f}(\vec{\xi})=f(\vec{\xi})$ almost ev. erywhere. Moreover $\hat{f}(\vec{\xi})$ is unique up to Borel null sets. Thus the following definition makes sense.

DEFINITION 1. If $F$ is a Wiener integrable function on $C[0, T]$, then by $\hat{E}[F(x-[x]+[\vec{\xi}\})]$ we mean any Borel measurable function of $\vec{\xi}$ which is equal to $E[F(x-[x]+[\vec{\xi}])]$ for a.e. $\vec{\xi}$ in $\mathbf{R}^{n}$.

We thus have the following useful formula, which is much simpler to apply than the inversion formula (1.4).

THEOREM 3. If $F$ is a Wiener integrable function on $C[0, T]$, then

$$
E\left(F(x) \mid X_{\tau}(x)=\vec{\xi}\right)=\hat{E}[F(x-[x]+[\vec{\xi}])] .
$$

In particular, if $F$ is also Borel measurable then

$$
E\left(F(x) \mid X_{\mathfrak{\tau}}(x)=\vec{\xi}\right)=E[F(x-[x]+[\vec{\xi}])] .
$$

The equality in (2.5) (and in (2.6)) means that both sides are Borel measurable functions of $\vec{\xi}$ and they are equal except for Borel null sets.

Proof. Equation (2.5) is obvious by the discussion following Theorem 2 and Definition 1 . If $F$ is also Borel measurable, then by the Fubini Theorem, $E[F(x-[x]+[\vec{\xi}])]$ is a Borel measurable function of $\vec{\xi}$, and hence (2.6) holds.

3. Examples. The following examples show that Theorem 3 above is indeed quite powerful and very simple to apply. have:

EXAMPLE 2. Let $F(x)=\int_{0}^{T} x(t) d t, x \in C[0, T]$. Then by (2.6) we

$$
\begin{aligned}
& E\left(\int_{0}^{T} x(t) d t \mid X_{\tau}(x)=\vec{\xi}\right)=E\left[\int_{0}^{T}(x(t)-[x](t)+[\vec{\xi}](t)) d t\right] \\
& \quad=\int_{0}^{T} E[x(t)-[x](t)+[\vec{\xi}](t)\} d t=\int_{0}^{T}[\vec{\xi}](t) d t \\
& \quad=\sum_{j=1}^{n} \int_{t_{j-1}}^{t_{j}}\left(\xi_{j-1}+\frac{t-t_{j-1}}{t_{j}-t_{j-1}}\left(\xi_{j}-\xi_{j-1}\right)\right) d t \\
& \quad=\frac{1}{2} \sum_{j=1}^{n}\left(\xi_{j}+\xi_{j-1}\right)\left(t_{j}-t_{j-1}\right) .
\end{aligned}
$$


In the above calculation, we used the Fubini Theorem to interchange the order of integration. In particular when $n=1$,

$$
E\left(\int_{0}^{T} x(t) d t \mid x(T)=\xi\right)=\frac{1}{2} \xi T
$$

which was computed by Yeh [9, pp. 629-631] using (1.4) with $n=1$; the computation was rather lengthy.

EXAMPLE 3. Let $F(x)=\int_{0}^{T}(x(t))^{2} d t, x \in C[0, T]$. Then, again by (2.6)

$$
\begin{aligned}
& E\left(\int_{0}^{T}(x(t))^{2} d t \mid X_{\tau}(x)=\vec{\xi}\right) \\
& \quad=E\left[\int_{0}^{T}(x(t)-[x](t)+[\vec{\xi}](t))^{2} d t\right] \\
& \quad=\int_{0}^{T} E\left[(x(t))^{2}+([x](t))^{2}+([\vec{\xi}](t))^{2}-2 x(t)[x](t)\right. \\
& +2 x(t)[\vec{\xi}](t)-2[x](t)[\vec{\xi}](t)] d t
\end{aligned}
$$

Using the properties $E[x(t)]=0$ and $E[x(s) x(t)]=\min \{s, t\}$ repeatedly, we obtain:

$$
\begin{aligned}
& E\left(\int_{0}^{T}(x(t))^{2} d t \mid X_{t}(x)=\vec{\xi}\right) \\
& \quad=\int_{0}^{T}\left\{t-\sum_{j=1}^{n} \chi_{\left[t_{j-1}, t_{j}\right]}(t)\left[t_{j-1}+\frac{\left(t-t_{j-1}\right)^{2}}{t_{j}-t_{j-1}}\right]+([\vec{\xi}](t))^{2}\right\} d t \\
& \quad=\frac{T^{2}}{2}-\frac{1}{3} \sum_{j=1}^{n}\left(t_{j}+2 t_{j-1}\right)\left(t_{j}-t_{j-1}\right) \\
& \quad+\frac{1}{3} \sum_{j=1}^{n}\left(\xi_{j}^{2}+\xi_{j} \xi_{j-1}+\xi_{j-1}^{2}\right)\left(t_{j}-t_{j-1}\right) .
\end{aligned}
$$

In particular when $n=1, E\left(\int_{0}^{T}(x(t))^{2} d t \mid x(T)=\xi\right)=T^{2} / 6+\xi^{2} T / 3$, which agrees with Yeh's computation [9, 631-632]. 
EXAMPLE 4. Let $F(x)=\exp \left\{\int_{0}^{T} x(t) d t\right\}, x \in C[0, T]$. Then

$$
\begin{aligned}
E\left(\exp \left\{\int_{0}^{T} x(t) d t\right\}\left\{X_{\tau}(x)=\vec{\xi}\right)\right. \\
=E\left[\exp \left\{\int_{0}^{T}(x(t)-[x](t)+[\vec{\xi}](t)) d t\right\}\right] \\
=\exp \left\{\int_{0}^{T}[\vec{\xi}](t) d t\right\} E\left[\exp \left\{\int_{0}^{T}(x(t)-[x](t)) d t\right\}\right]
\end{aligned}
$$

It is interesting to note that for each fixed $u \in C[0, T]$, we have, as expected,

$$
\lim _{\|\tau\| \rightarrow 0} E\left(\exp \left\{\int_{0}^{T} x(t) d t\right\}\left\{X_{\tau}(x)=X_{\tau}(u)\right)=\exp \left\{\int_{0}^{T} u(t) d t\right\} .\right.
$$

Some of the above examples are also considered by Chang and Chang [3] using the inversion formula (1.4); their computations are very complicated.

4. A generalization of the Kac-Feynman formula. Under the assumption that $V$ is a nonnegative continuous function on $\mathbf{R}$ satisfying the condition

$$
\int_{\mathbf{R}} V(\xi) e^{-\xi^{2} / 2 t} d \xi<\infty \quad \text { for every } t>0
$$

Yeh [9] has shown that the function $U$ defined on $\mathbf{R} \times(0, \infty)$ by

$$
\begin{gathered}
U(\xi, t)=E\left(\exp \left\{-\int_{0}^{t} V(x(s)) d s\right\} \mid x(t)=\xi\right) \\
\cdot(2 \pi t)^{-1 / 2} \exp \left(-\frac{\xi^{2}}{2 t}\right)
\end{gathered}
$$

satisfies the Kac-Feynman integral equation

$$
\begin{aligned}
U(\xi, t)=\frac{1}{\sqrt{2 \pi t}} e^{-\xi^{2} / 2 t} & -\int_{0}^{t}\left[\frac{1}{\sqrt{2 \pi(t-s)}} \int_{-\infty}^{\infty} \exp \left\{-\frac{(\xi-\eta)^{2}}{2(t-s)}\right\}\right. \\
& \cdot V(\eta) U(\eta, s) d \eta] d s,
\end{aligned}
$$

whose solution is given by

$$
U(\xi, t)=\sum_{k=0}^{\infty}(-1)^{k} U_{k}(\xi, t), \quad(\xi, t) \in \mathbf{R} \times(0, \infty) .
$$


where the sequence $\left\{U_{k}\right\}$ is defined inductively by

$$
U_{0}(\xi, t)=\frac{1}{\sqrt{2 \pi t}} e^{-\xi^{2} / 2 t},
$$

$U_{k+1}(\xi, t)$

$$
=\int_{0}^{t}\left(\frac{1}{\sqrt{2 \pi(t-s)}} \int_{-\infty}^{\infty} \exp \left\{-\frac{(\xi-\eta)^{2}}{2(t-s)}\right\} V(\eta) U_{k}(\eta, s) d \eta\right) d s
$$

for $k=0,1,2, \ldots$ Thus $E\left(\exp \left\{-\int_{0}^{t} V(x(s)) d s\right\} \mid x(t)=\xi\right)$ can be found using (4.1).

Now, under the same assumptions of $V$, using Theorem 3, we proceed to find the multi-conditional expectation

$$
E\left(\exp \left\{-\int_{0}^{t} V(x(s)) d s\right\} \mid x\left(t_{i}\right)=\xi_{i}, i=1, \ldots, n\right),
$$

where $0<t_{1}<t_{2}<\cdots<t_{n}=t$. First consider the conditional expectation for $0<t_{1}<t$,

$$
I \equiv E\left(\exp \left\{-\int_{t_{1}}^{t} V(x(s)) d s\right\} \mid x\left(t_{1}\right)=\xi_{1}, x(t)=\xi\right) .
$$

Then, by Theorem 3, (2.1) and (2.2),

$$
\begin{array}{r}
I=E\left[\operatorname { e x p } \left\{-\int_{t_{1}}^{t} V\left(x(s)-x\left(t_{1}\right)-\frac{s-t_{1}}{t-t_{1}}\left(x(t)-x\left(t_{1}\right)\right)\right.\right.\right. \\
\left.\left.\left.+\xi_{1}+\frac{s-t_{1}}{t-t_{1}}\left(\xi-\xi_{1}\right)\right) d s\right\}\right] .
\end{array}
$$

Since the Brownian motion $x(t)$ has stationary increments, it follows from (4.4) that

$$
\begin{aligned}
I & =E\left[\operatorname { e x p } \left\{-\int_{0}^{t-t_{1}} V\left(x(u)-\frac{u}{t-t_{1}}\left(x\left(t-t_{1}\right)\right)+\xi_{1}\right.\right.\right. \\
& \left.\left.\left.+\frac{u}{t-t_{1}}\left(\xi-\xi_{1}\right)\right) d u\right\}\right] \\
& =E\left(\exp \left\{-\int_{0}^{t-t_{1}} V\left(x(u)+\xi_{1}\right) d u\right\} \mid x\left(t-t_{1}\right)=\xi-\xi_{1}\right) \\
& =E\left(\exp \left\{-\int_{0}^{t-t_{1}} W(x(u)) d u\right\} \mid x\left(t-t_{1}\right)=\xi-\xi_{1}\right),
\end{aligned}
$$

where $W(x(u))=V\left(x(u)+\xi_{1}\right)$. Next, let

$$
U\left(\xi_{1}, \xi-\xi_{1}, t-t_{1}\right)=I \cdot\left[2 \pi\left(t-t_{1}\right)\right]^{-1 / 2} \exp \left\{-\frac{\left(\xi-\xi_{1}\right)^{2}}{2\left(t-t_{1}\right)}\right\} .
$$


Then, applying (4.1) and (4.2) to (4.5), we obtain that

$$
\begin{aligned}
U\left(\xi_{1}, \xi-\xi_{1}, t-t_{1}\right) & \\
= & {\left[2 \pi\left(t-t_{1}\right)\right]^{-1 / 2} \exp \left\{-\frac{\left(\xi-\xi_{1}\right)^{2}}{2\left(t-t_{1}\right)}\right\} } \\
& -\int_{0}^{t-t_{1}}\left[\left[2 \pi\left(t-t_{1}-u\right)\right]^{-1 / 2}\right. \\
& \left.\cdot \int_{-\infty}^{\infty} \exp \left\{-\frac{\left(\xi-\xi_{1}-\eta\right)^{2}}{2\left(t-t_{1}-u\right)}\right\} W(\eta) U\left(\xi_{1}, \eta, u\right) d \eta\right] d u .
\end{aligned}
$$

Since $W(\eta)=V\left(\eta+\xi_{1}\right)$, we may use the transformation $\gamma=\eta+\xi_{1}$ to obtain

$$
\begin{aligned}
& U\left(\xi_{1}, \xi-\xi_{1}, t-t_{1}\right) \\
& =\left[2 \pi\left(t-t_{1}\right)\right]^{-1 / 2} \exp \left\{-\frac{\left(\xi-\xi_{1}\right)^{2}}{2\left(t-t_{1}\right)}\right\} \\
& -\int_{0}^{t-t_{1}}\left[\left[2 \pi\left(t-t_{1}-u\right)\right]^{-1 / 2}\right. \\
& \left.\quad \cdot \int_{-\infty}^{\infty} \exp \left\{-\frac{(\xi-\gamma)^{2}}{2\left(t-t_{1}-u\right)}\right\} V(\gamma) U\left(\xi_{1}, \gamma-\xi_{1}, u\right) d \gamma\right] d u .
\end{aligned}
$$

But this equation is similar to the Kac-Feynman integral equation, and hence its solution is given by

$$
U\left(\xi_{1}, \xi-\xi_{1}, t-t_{1}\right)=\sum_{k=0}^{\infty}(-1)^{k} U_{k}\left(\xi, \xi-\xi_{1}, t-t_{1}\right),
$$

where

$$
U_{0}\left(\xi_{1}, \xi-\xi_{1}, t-t_{1}\right)=\left[2 \pi\left(t-t_{1}-u\right)\right]^{-1 / 2} \exp \left\{-\frac{\left(\xi-\xi_{1}\right)^{2}}{2\left(t-t_{1}\right)}\right\},
$$

and

$$
\begin{aligned}
& U_{k+1}\left(\xi_{1}, \xi-\xi_{1}, t-t_{1}\right) \\
& =\int_{0}^{t-t_{1}}\left[\left[2 \pi\left(t-t_{1}-u\right)\right]^{-1 / 2} \int_{-\infty}^{\infty} \exp \left\{-\frac{(\xi-\gamma)^{2}}{2\left(t-t_{1}-u\right)}\right\}\right. \\
& \left.\cdot V(\gamma) U_{k}\left(\xi_{1}, \gamma-\xi_{1}, u\right) d \gamma\right] d u
\end{aligned}
$$

for $k=0,1,2, \ldots$ In particular the solution $U(\xi, t)$ of the KacFeynman integral equation (4.2) is a special case, namely

$$
U(\xi, t)=U(0, \xi, t) \text {. }
$$


We are now ready to write out an expression for the multi-conditional expectation. Using the Markov property of the conditional process and the multiplicative nature of the exponential function, we may write

$$
\begin{aligned}
& E\left(\exp \left\{-\int_{0}^{t} V(x(s)) d s\right\} \mid x\left(t_{i}\right)=\xi_{i}, i=1,2, \ldots, n\right) \\
& =\prod_{i=1}^{N} E\left(\exp \left\{-\int_{t_{r-1}}^{t_{i}} V(x(s)) d s\right\} \mid x\left(t_{i-1}\right)=\xi_{i-1}, x\left(t_{i}\right)=\xi_{i}\right) \\
& =\prod_{i=1}^{N}\left[2 \pi\left(t_{i}-t_{i-1}\right)\right]^{1 / 2} \exp \left\{\frac{\left(\xi_{i}-\xi_{i-1}\right)^{2}}{2\left(t_{i}-t_{i-1}\right)}\right\} \\
& \cdot U\left(\xi_{i-1}, \xi_{i}-\xi_{i-1}, t_{i}-t_{i-1}\right) .
\end{aligned}
$$

Also, we have

$$
\begin{array}{r}
\text { (4.7)E }\left(\exp \left\{-\int_{0}^{t} V(x(s)) d s\right\} \mid a_{i}<x\left(t_{i}\right)<b_{i}, i=1,2, \ldots, n\right) \\
=\int_{a_{n}}^{b_{n}} \cdots \int_{a_{1}}^{b_{1}} \prod_{i=1}^{n} U\left(\xi_{i-1}, \xi_{i}-\xi_{i-1}, t_{i}-t_{i-1}\right) d \xi_{1} \cdots d \xi_{n}
\end{array}
$$

5. Translation of multi-conditional Wiener integrals. The CameronMartin Translation Theorem [2, 7] states that if $x_{0}(t)=\int_{0}^{t} h(s) d s$ for all $t \in[0, T]$ with $h \in L^{2}[0, T]$, and if $T_{1}$ is the transformation of $C[0, T]$ into itself defined by

$$
T_{1}(x)=x+x_{0} \quad \text { for } x \in C[0, T],
$$

then for any Wiener integrable function $F$ on $C[0, T]$ and any Wiener measurable set $\Gamma$

$$
\int_{\Gamma} F(y) m_{w}(d y)=\int_{T_{1}^{-1}(\Gamma)} F\left(x+x_{0}\right) J\left(x_{0}, x\right) m_{w}(d x)
$$

where

$$
J\left(x_{0}, x\right)=\exp \left\{-\frac{1}{2} \int_{0}^{T}(h(t))^{2} d t\right\} \exp \left\{-\int_{0}^{T} h(t) d x(t)\right\}
$$

and $\int_{0}^{T} h(t) d x(t)$ is a stochastic integral with mean zero, and variance $\int_{0}^{T}(h(t))^{2} d t$. In particular, if $\Gamma=C[0, T]$, then (5.1) becomes:

$$
E[F(y)]=E\left[F\left(x+x_{0}\right) J\left(x_{0}, x\right)\right]
$$


Yeh [10] gives a conditional version of (5.3) which states that under the same conditions as above,

$$
\begin{aligned}
E(F(y) \mid y(T)=\xi)= & E\left(F\left(x+x_{0}\right) J\left(x_{0}, x\right) \mid x(T)=\xi-x_{0}(T)\right) \\
& \cdot \exp \left\{-\frac{x_{0}^{2}(T)}{2 T}\right\} \exp \left\{\frac{x_{0}(T)}{T} \xi\right\} .
\end{aligned}
$$

The following is the multi-conditional version of (5.3). Its proof is very simple and straightforward because our "inversion formula", Theorem 3 , is so simple and easy to apply.

THEOREM 4. Let $x_{0}(t)=\int_{0}^{t} h(s) d s$ for all $t \in[0, T]$ with $h \in$ $L^{2}[0, T]$. If $F$ is any Wiener integrable function on $C[0, T]$, then for any partition $\tau=\left\{t_{1}, \ldots, t_{n}\right\}$ of $[0, T]$,

$$
\begin{aligned}
& E\left(F(y) \mid y\left(t_{j}\right)=\xi_{j}, j=1, \ldots, n\right) \\
& =E\left(F\left(x+x_{0}\right) J\left(x_{0}, x\right) \mid x\left(t_{j}\right)=\xi_{j}-x_{0}\left(t_{j}\right), j=1, \ldots, n\right) \\
& \quad \cdot \prod_{j=1}^{n} \exp \left\{\frac{-\left(x_{0}\left(t_{j}\right)-x_{0}\left(t_{j-1}\right)\right)^{2}}{2\left(t_{j}-t_{j-1}\right)}+\frac{\left(x_{0}\left(t_{j}\right)-x_{0}\left(t_{j-1}\right)\right)\left(\xi_{j}-\xi_{j-1}\right)}{t_{j}-t_{j-1}}\right\}
\end{aligned}
$$

where $J\left(x_{0}, x\right)$ is given by (5.2).

Proof. By use of Theorem 3 we see that

$$
E\left(F(y) \mid y\left(t_{j}\right)=\xi_{j}, j=1, \ldots, n\right)=\hat{E}[F(y-[y]+[\vec{\xi}])] .
$$

Now applying (5.3) and noting that $\left[x+x_{0}\right](t)=[x](t)+\left[x_{0}\right](t)$ we have

(5.6) $\hat{E}[F(y-[y]+[\vec{\xi}])]=\hat{E}\left[F\left(x+x_{0}-[x]-\left[x_{0}\right]+[\vec{\xi}]\right) J\left(x_{0}, x\right)\right]$.

Next we rewrite $J\left(x_{0}, x\right)$ in the form

$$
\text { (5.7) } \begin{aligned}
J\left(x_{0} x,\right)= & \exp \left\{-\frac{1}{2} \int_{0}^{T}(h(t))^{2} d t\right\} \\
& \cdot \exp \left\{-\int_{0}^{T} h(t) d\left(x(t)-[x](t)+[\vec{\xi}](t)-\left[x_{0}\right](t)\right)\right\} \\
& \cdot \exp \left\{-\int_{0}^{T} h(t) d[x](t)\right\} \\
& \cdot \exp \left\{\int_{0}^{T} h(t) d\left([\vec{\xi}](t)-\left[x_{0}\right](t)\right)\right\}
\end{aligned}
$$


But

(5.8)

$$
\begin{aligned}
& \int_{0}^{T} h(t) d[x](t) \\
& \quad=\sum_{j=1}^{n} \int_{t_{j-1}}^{t_{j}} h(t) d\left(x\left(t_{j-1}\right)+\frac{t-t_{j-1}}{t_{j}-t_{j-1}}\left(x\left(t_{j}\right)-x\left(t_{j-1}\right)\right)\right) \\
& \quad=\sum_{j=1}^{n} \frac{x_{0}\left(t_{j}\right)-x_{0}\left(t_{j-1}\right)}{t_{j}-t_{j-1}}\left(x\left(t_{j}\right)-x\left(t_{j-1}\right)\right)
\end{aligned}
$$

Similarly,

$$
\begin{array}{rl}
\int_{0}^{T} & h(t) d\left([\vec{\xi}](t)-\left[x_{0}\right](t)\right) \\
& =\sum_{j=1}^{n} \frac{x_{0}\left(t_{j}\right)-x_{0}\left(t_{j-1}\right)}{t_{j}-t_{j-1}}\left[\left(\xi_{j}-\xi_{j-1}\right)-\left(x_{0}\left(t_{j}\right)-x_{0}\left(t_{j-1}\right)\right)\right]
\end{array}
$$

On the other hand,

$$
\begin{aligned}
& E\left[\exp \left\{-\int_{0}^{T} h(t) d[x](t)\right\}\right] \\
& \quad=E\left[\exp \left\{-\sum_{j=1}^{n} \frac{x_{0}\left(t_{j}\right)-x_{0}\left(t_{j-1}\right)}{\sqrt{t_{j}-t_{j-1}}} \cdot \frac{x\left(t_{j}\right)-x\left(t_{j-1}\right)}{\sqrt{t_{j}-t_{j-1}}}\right\}\right] .
\end{aligned}
$$

Since

$$
\left\{\frac{x\left(t_{j}\right)-x\left(t_{j-1}\right)}{\sqrt{t_{j}-t_{j-1}}}, j=1, \ldots, n\right\}
$$

is a set of independent identically distributed standard normal random variables, we have that

$$
\begin{aligned}
E[\exp & \left.\left\{-\int_{0}^{T} h(t) d[x](t)\right\}\right] \\
& =\prod_{j=1}^{n} \exp \left\{\frac{\left(x_{0}\left(t_{j}\right)-x_{0}\left(t_{j-1}\right)\right)^{2}}{2\left(t_{j}-t_{j-1}\right)}\right\} .
\end{aligned}
$$

Now using the expression (5.8), one can easily see that $\int_{0}^{T} h(t) d[x](t)$ is independent of the process $\{x(t)-[x](t): 0 \leq t \leq T\}$. Thus,

$$
\begin{aligned}
F\left(x+x_{0}-[x]-\left[x_{0}\right]+[\vec{\xi}]\right) \\
\cdot \exp \left\{-\int_{0}^{T} h(t) d\left(x(t)-[x](t)+[\vec{\xi}](t)-\left[x_{0}\right](t)\right)\right\}
\end{aligned}
$$


and $\exp \left\{-\int_{0}^{T} h(t) d[x](t)\right\}$ are independent. Hence, using (5.7), (5.9), and $(5.10)$, it follows that

$$
\begin{aligned}
\hat{E}\left[F\left(x+x_{0}-[x]-\left[x_{0}\right]+[\vec{\xi}]\right) J\left(x_{0}, x\right)\right] \\
=\hat{E}\left[F\left(x+x_{0}-[x]-\left[x_{0}\right]+[\vec{\xi}]\right)\right. \\
\left.\cdot \exp \left\{-\int_{0}^{T} h(t) d\left(x(t)-[x](t)+[\vec{\xi}](t)-\left[x_{0}\right](t)\right)\right\}\right] \\
\cdot \exp \left\{-\frac{1}{2} \int_{0}^{T}(h(t))^{2} d t\right\} \prod_{j=1}^{n} \exp \left\{\frac{\left(x_{0}\left(t_{j}\right)-x_{0}\left(t_{j-1}\right)\right)^{2}}{2\left(t_{j}-t_{j-1}\right)}\right\} \\
\cdot \prod_{j=1}^{n} \exp \left\{\frac{x_{0}\left(t_{j}\right)-x_{0}\left(t_{j-1}\right)}{t_{j}-t_{j-1}}\left[\left(\xi_{j}-\xi_{j-1}\right)-\left(x_{0}\left(t_{j}\right)-x_{0}\left(t_{j-1}\right)\right)\right]\right\} .
\end{aligned}
$$

Therefore, by using (2.5)

$$
\begin{aligned}
\hat{E}\left[F\left(x+x_{0}-\{x]-\left[x_{0}\right]+[\vec{\xi}]\right) J\left(x_{0}, x\right)\right] & j=1, \ldots, n) \\
= & E\left(F\left(x+x_{0}\right) \exp \left\{-\int_{0}^{T} h(t) d x(t)\right\} \mid x\left(t_{j}\right)=\xi_{j}-x_{0}\left(t_{j}\right),\right. \\
& \cdot \exp \left\{-\frac{1}{2} \int_{0}^{T}(h(t))^{2} d t\right\} \\
& \cdot \prod_{j=1}^{n} \exp \left\{-\frac{\left(x_{0}\left(t_{j}\right)-x_{0}\left(t_{j-1}\right)\right)^{2}}{2\left(t_{j}-t_{j-1}\right)}+\frac{\left(x_{0}\left(t_{j}\right)-x_{0}\left(t_{j-1}\right)\right)\left(\xi_{j}-\xi_{j-1}\right)}{t_{j}-t_{j-1}}\right\} .
\end{aligned}
$$

This, together with (5.6) and (5.5), completes the proof.

\section{REFERENCES}

[1] L. Breiman, Probability, Addison-Wesley, Reading, Mass. 1968.

[2] R. H. Cameron and W. T. Martin, Transformation of Wiener integrals under translations, Ann. Math., 45 (1944), 386-396.

[3] K. S. Chang and J. S. Chang, Evaluation of some conditional Wiener integrals, Bull. Korean Math. Soc., 21 (1984), 99-106.

[4] J. L. Doob, Stochastic Processes, Wiley, New York, 1953. 
[5] M. Kac, Probability and Related Topics in Physical Sciences, Interscience Publishers, New York, 1959.

[6] H. G. Tucker, A Graduate Course in Probability, Academic Press, New York, 1967.

[7] J. Yeh, Stochastic Processes and the Wiener Integral, Marcel Dekker, New York, 1973.

[8] , Inversion of conditional expectations, Pacific J. Math., 52 (1974), 631640.

[9] _. Inversion of conditional Wiener integrals, Pacific J. Math., 59 (1975), 623-638.

[10] - Transformation of conditional Wiener integrals under translation and the Cameron-Martin translation theorem, Tôhoku Math. J., 30 (1978), 505-515.

Received May 24, 1987 and in revised form November 18, 1987.

MiAMI UNIVERSITY

OXFORD, OH 45056

AND

UNIVERSITY OF NEBRASKA

LINCOLN, NE 68588-0323 


\section{PACIFIC JOURNAL OF MATHEMATICS \\ EDITORS}

\author{
V. S. VARADARAJAN \\ (Managing Editor) \\ University of California \\ Los Angeles, CA 90024 \\ HERBERT CLEMENS \\ University of Utah \\ Salt Lake City, UT 84112 \\ R. FINN \\ Stanford University \\ Stanford, CA 94305
}

\author{
HERMANN FLASCHKA \\ University of Arizona \\ Tucson, AZ 85721
}

vAUGHAN F. R. JONES

University of California

Berkeley, CA 94720

STEVENKERCKH OFF

Stanford University

Stanford, CA 94305

\author{
ROBION KIRBY \\ University of California \\ Berkeley, CA 94720 \\ C. C. MOORE \\ University of California \\ Berkeley, CA 94720 \\ HAROLD T ARK
}

University of California, San Diego La Jolla, CA 92093

\section{ASSOCIATE EDITORS}
R. ARENS
E. F. BECKENBACH
B. H. NEUMANN
F. WOLF
K. YOSHIDA

(1906-1982)

\section{SUPPORTING INSTITUTIONS}

\author{
UNIVERSITY OF ARIZONA \\ UNIVERSITY OF BRITISH COLUMBIA \\ CALIFORNIA INSTITUTE OF TECHNOLOGY \\ UNIVERSITY OF CALIFORNIA \\ MONTANA STATE UNIVERSITY \\ UNIVERSITY OF NEVADA, RENO \\ NEW MEXICO STATE UNIVERSITY \\ OREGON STATE UNIVERSITY
}

\author{
UNIVERSITY OF OREGON \\ UNIVERSITY OF SOUTHERN CALIFORNIA \\ STANFORD UNIVERSITY \\ UNIVERSITY OF HAWAII \\ UNIVERSITY OF TOKYO \\ UNIVERSITY OF UTAH \\ WASHINGTON STATE UNIVERSITY \\ UNIVERSITY OF WASHINGTON
}

The Supporting Institutions listed above contribute to the cost of publication of this Journal, but they are not owners or publishers and have no responsibility for its content or policies.

Mathematical papers intended for publication in the Pacific Journal of Mathematics should be in typed form or offset-reproduced (not dittoed), double spaced with large margins. Please do not use built up fractions in the text of the manuscript. However, you may use them in the displayed equations. Underline Greek letters in red, German in green, and script in blue. The first paragraph must be capable of being used separately as a synopsis of the entire paper. In particular it should contain no bibliographic references. Please propose a heading for the odd numbered pages of less than 35 characters. Manuscripts, in triplicate, may be sent to any one of the editors. Please classify according to the scheme of Math. Reviews, Index to Vol. 39. Supply name and address of author to whom proofs should be sent. All other communications should be addressed to the managing editor, or Elaine Barth, University of California, Los Angeles, California 90024.

There are page-charges associated with articles appearing in the Pacific Journal of Mathematics. These charges are expected to be paid by the author's University, Government Agency or Company. If the author or authors do not have access to such Institutional support these charges are waived. Single authors will receive 50 free reprints; joint authors will receive a total of 100 free reprints. Additional copies may be obtained at cost in multiples of 50 .

The Pacific Journal of Mathematics is issued monthly as of January 1966. Regular subscription rate: $\$ 190.00$ a year (5 Vols., 10 issues). Special rate: $\$ 95.00$ a year to individual members of supporting institutions.

Subscriptions, orders for numbers issued in the last three calendar years, and changes of address should be sent to Pacific Journal of Mathematics, P.O. Box 969, Carmel Valley, CA 93924, U.S.A. Old back numbers obtainable from Kraus Periodicals Co., Route 100, Millwood, NY 10546.

The Pacific Journal of Mathematics at P.O. Box 969, Carmel Valley, CA 93924 (ISSN 0030-8730) publishes 5 volumes per year. Application to mail at Second-class postage rates is pending at Carmel Valley, California, and additional mailing offices. Postmaster: send address changes to Pacific Journal of Mathematics, P.O. Box 969, Carmel Valley, CA 93924.

\section{PUBLISHED BY PACIFIC JOURNAL OF MATHEMATICS, A NON-PROFIT CORPORATION} Copyright (C) 1988 by Pacific Journal of Mathematics 


\section{Pacific Journal of Mathematics}

Vol. 135, No. $2 \quad$ October, 1988

Waleed A. Al-Salam and Mourad Ismail, $q$-beta integrals and the

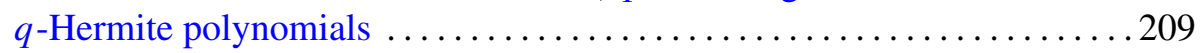

Johnny E. Brown, On the Ilieff-Sendov conjecture $\ldots \ldots \ldots \ldots \ldots \ldots \ldots 223$

Lawrence Jay Corwin and Frederick Paul Greenleaf, Spectrum and

multiplicities for restrictions of unitary representations in nilpotent Lie

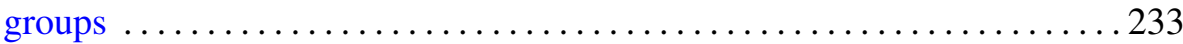

Robert Jay Daverman, 1-dimensional phenomena in cell-like mappings on 3-manifolds .......................................... 269

P. D. T. A. Elliott, A localized Erdős-Wintner theorem .............. 287

Richard John Gardner, Relative width measures and the plank problem . . . 299

F. Garibay, Peter Abraham Greenberg, L. Reséndis and Juan José

Rivaud, The geometry of sum-preserving permutations ............313

Shanyu Ji, Uniqueness problem without multiplicities in value distribution

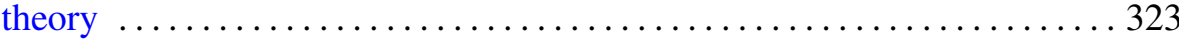

Igal Megory-Cohen, Finite-dimensional representation of classical

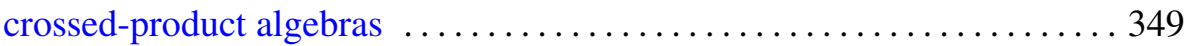

Mirko Navara, Pavel Pták and Vladimír Rogalewicz, Enlargements of

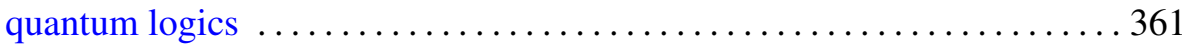

Claudio Nebbia, Amenability and Kunze-Stein property for groups acting

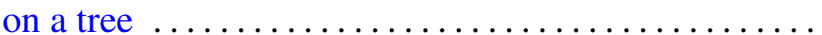

Chull Park and David Lee Skoug, A simple formula for conditional Wiener

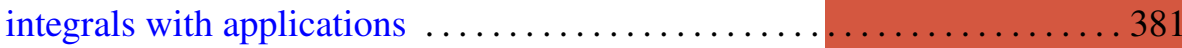

Ronald Scott Irving and Brad Shelton, Correction to: "Loewy series and simple projective modules in the category $\mathrm{O}_{s} " \ldots .$.

Robert Tijdeman and Lian Xiang Wang, Correction to: "Sums of products

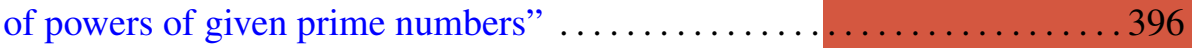

